\title{
LA CASCARILLA DE LA SOJA: POTENCIAL COMPONENTE DE ALIMENTOS FUNCIONALES
}

\section{SOYBEAN HULL: POTENTIAL INGREDIENTS FOR FUNCTIONAL FOODS}

\begin{abstract}
María Catalina Olguin B. (1), Marta Delia Posadas R. (2), Gilda Celina Revelant Z. (1), Verónica Labourdette P. (2), Darío Marinozzi T. (1), Héctor Daniel Elías M. (1), María del Carmen Gayol F. (2), María Isabel Zingale V. (1)
\end{abstract}

(1) Cátedra de Bromatología. Facultad de Ciencias Bioquímicas y Farmacéuticas, Universidad Nacional de Rosario, Argentina

(2) Cátedra de Biología. Facultad de Ciencias Médicas, Universidad Nacional de Rosario, Argentina

\begin{abstract}
The effects of soybean bran, a residue of soybean oil extraction, rich in dietary fiber-both soluble and insoluble - on fat distribution, plasmatic and liver lipids, glycemia and glycemic load, were studied on adult male obese diabetic $\beta$ rats. Two hundred days old $\beta$ rats were fed two diets with $15 \%$ sodium caseinate as protein source and $10 \%$ dietary fiber from soybean bran $(S)$ or cellulose $(C)$ during 30 and 60 days. Significant diminutions in blood cholesterol levels were registered in group $S$ on day 30 as well as on day 60 . Diet $S$ significantly attenuated the characteristic increase in blood triacylglycerols levels and the usual progressive increase in blood glucose levels expressed in this line of rats. Diet $S$ decreased significantly liver total lipids, cholesterol and triacylglycerols compared with $C$. No differences were registered between groups neither in food intake nor in biomass. These effects are attributed to the combined effects of the soluble and insoluble fiber fractions present in soybean bran. In conclusion, soybean bran may be considered as a useful component of functional foods designed for human nutrition.
\end{abstract}

Key words: soybean bran; functional foods; obesity.

Este trabajo fue recibido el 9 de Septiembre de 2008 y aceptado para ser publicado el 20 de Junio de 2009.

\section{INTRODUCCIÓN}

Basándose en evidencias científicas cada vez más numerosas, que prueban la eficacia de los buenos hábitos de vida para prevenir la instalación del sobrepeso, la obesidad y sus derivaciones metabólicas $(1,2)$, organismos internacionales tales como FAO y OMS coinciden en resaltar la importancia de realizar una alimentación armónica y disminuir la ingesta energética $(3,4)$.

En tal sentido, diversos estudios epidemiológicos y clínicos han comprobado el efecto beneficioso sobre el peso corporal y el perfil glucolipídico que ocasiona el consumo de alimentos ricos en fibra, ya sean de origen vegetal en forma natural o como productos alimenticios funcionales (5-7).

Dada la baja proporción de hidratos de carbono disponibles, la notable riqueza proteica y la cantidad y el tipo de fibra que contienen (8-10), entre los vegetales se recomienda particularmente el consumo de leguminosas, tales como la soja, rica en fibra tanto soluble como insoluble, que ha demostrado ser eficiente en el control y prevención de enfermedades crónicas no transmisibles como la diabetes tipo 2, patología cardiovascular y el cáncer de colon $(11,12)$. La fibra de soja purificada o como componente de dietas experimentales ha sido ensayada en diferentes modelos animales, en los que se han constatado efectos moderadores de la concentración de colesterol sérico y hepático (13). Ensayos en humanos, de mediano y largo plazo también han dado lugar a efectos de disminución de colesterol y triacilgliceroles séricos (14).

En Argentina, uno de los cultivos de mayor importancia económica es la soja; su producción se destina mayoritariamente a la obtención de aceite el que se exporta y parte de la harina o expeller remanente del proceso 
de extracción, se emplea en alimentación humana. El salvado, pericarpio o cascarilla de las semillas de soja suele destinarse, como en otros países, a suplementar raciones para el ganado vacuno, con buena eficiencia, comparable a la del maíz; como fuente energética (15). Sumado a esto, la alta proporción y el tipo de fibra presente en el salvado de la soja, dan lugar a una ventajosa fermentabilidad ruminal cuando se lo combina con el forraje tradicional (16).

Vista la riqueza en fibra del salvado de la soja y los resultados beneficiosos obtenidos con el consumo de otras fracciones de sus semillas sobre el perfil glucolipídico y la distribución del tejido adiposo de ratas IIMbBeta con obesidad y diabetes tipo 2 (17 -19), el objetivo de este estudio fue evaluar los efectos del salvado de la soja sobre variables relacionadas con esas patologías en la mencionada cepa de ratas, obtenida por desadaptación genético ambiental y alto grado de endocría (20).

El objetivo planteado surge del interés que despierta la posibilidad de emplear la cascarilla de soja como componente de un alimento funcional para adultos con sobrepeso u obesidad. El alimento funcional podría revestir la forma de panificados, considerando el consumo habitual de estos productos y su bajo costo; también se podría adicionar el salvado a yogures, sopas, o emplearse como ligante para la preparación de hamburguesas (21, 22). De este modo se otorgaría un valor adicional a esta fracción del grano de soja.

\section{MATERIALES Y MÉTODO Unidades experimentales}

Ratas macho de la cepa IIMb/ $\beta$ de 200 días de edad $(n=32)$, criadas en el Bioterio de la Cátedra de Biología de la Facultad de Ciencias Médicas, alojadas durante los experimentos en jaulas individuales, alimentadas ad libi- tum, sometidas a períodos de luz-oscuridad de 12 horas y con temperatura ambiental regulada a $22 \pm 2^{\circ} \mathrm{C}$.

\section{Tratamientos}

Se administraron durante 60 días, dos dietas cuya composición se detalla en la tabla 1 . El salvado o cascarilla de la soja fue obtenido por descascarado del grano seco, tal como se realiza en la etapa previa a la molienda para la extracción del aceite, en la industria aceitera argentina. El salvado obtenido se sometió a un tratamiento con vapor de agua $\left(105-110^{\circ} \mathrm{C}\right)$, se secó con corriente de aire $\left(70-80^{\circ} \mathrm{C}\right)$ hasta una humedad no superior al $3,5 \%$ y se molió a granulometría $0,30-0,5 \mathrm{~mm}$. El tratamiento con vapor de agua asegura la inactivación de los compuestos antinutricionales que pudieran estar presentes en esta fracción de la leguminosa (23). La soja provino de un cultivo orgánico de la localidad de Pergamino, provincia de Buenos Aires, Argentina.

\section{Registros y mediciones}

Se efectuó el registro de la ingestión de alimento y del peso corporal de los animales cada dos días.

Al inicio, al día 30 y al día 60, se extrajeron muestras de sangre, previo ayuno nocturno de 12 horas, para el dosaje de glicemia, colesterolemia total y triacilglicerolemia.

En los días 30 y 60 se realizó una prueba de tolerancia a la glucosa (perfundiendo por sonda gástrica una solución glucosada $10 \mathrm{~g} / \mathrm{dl}$ a razón de $200 \mathrm{mg} / 100 \mathrm{~g}$ de peso).

El día 30 se sacrificaron 9 animales de cada grupo. El experimento se continuó con los 14 animales restantes que se sacrificaron en el día 60. Se disecaron y pesaron los hígados, los panículos adiposos retroperitoneales y perigonadales. En los hígados se determinaron lípidos

\section{TABLA 1}

\section{Composición de las dietas (g)}

\section{Dieta C}

Caseinato de sodio (88,3 g\% de proteínas)

Mezcla mineral según AIN 93

Mezcla vitamínica según AIN 93

Aceite de girasol

Celulosa microcristalina

Salvado de soja

Cloruro de colina

Almidón de maíz csp
10

10

--

0,15

100

\section{Dieta S}

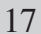

3

1

10

15

0,15

100 
totales, colesterol y triacilgliceroles.

El estudio fue aprobado por el Comité de Bioética de la Facultad de Ciencias Médicas.

\section{Métodos analíticos}

Para estudiar la composición proximal del salvado de la soja y de las dietas formuladas se emplearon los siguientes métodos (24):

- Pérdida de peso a $105^{\circ} \mathrm{C}$, (AOAC 1990, método 945.39) para la determinación de humedad.

- Extracto etéreo en Soxhlet, (AOAC 1990, método 920.85) para el dosaje de materia grasa.

- Calcinación en mufla a $550^{\circ} \mathrm{C}$, (AOAC 1990, método 923.03) para la cuantificación del contenido mineral.

- Método de Kjeldahl (AOAC 1990, 984.13) para el dosaje de proteína cruda. Factores empleados: para caseína 6,38; para proteínas de soja 5,75.

- Método enzimático-gravimétrico, (AOAC 1990, 993.19) para la cuantificación de fibra dietaria total, soluble e insoluble.

- Método de Thompson y Erdman para la determinación de ácido fítico (25)

- Métodos enzimático-espectrofotométricos (Wiener Lab., Argentina) para las determinaciones plasmáticas y hepáticas.

- Método de Folch et al (26) para la extracción de los lípidos de hígado y panículos adiposos.

\section{Análisis estadístico}

Los resultados se expresan como media \pm desvío estándar. Se aplicaron, según se consideró conveniente, las pruebas estadísticas: análisis de variancia y post test de Tukey o t de Student, del paquete estadístico Minitab Release 12. Las diferencias se consideraron significativas cuando fue $\mathrm{p}<0.05$.

\section{RESULTADOS}

En la composición proximal se comprobó un alto contenido de fibra dietaria en el salvado de la soja, detectándose la presencia de ambas fracciones: insoluble y soluble (tabla 2).

Se constató una significativa disminución en los niveles de colesterol sérico total en el grupo alimentado con la dieta con salvado de soja respecto del grupo C tanto a los 30 como a los 60 días del experimento. En el grupo $\mathrm{S}$ esta variable se mantuvo con escasas variaciones mientras que el grupo $\mathrm{C}$ fue incrementando de modo significativo aunque dentro de los valores normales para estos animales, el nivel de colesterolemia con respecto al inicial (tabla 3).

Los triacilgliceroles iniciales elevados, característicos de la línea IIMb/ $\beta$, se mantuvieron sin variaciones significativas a lo largo del experimento en el caso del grupo $\mathrm{S}$ y aumentaron progresiva y significativamente en el grupo C (tabla 3).

No se registraron diferencias significativas por efectos de la dieta ni del tiempo de tratamiento en la glucemia basal ni tras 120 minutos de sobrecarga con glucosa. La interacción de ambos factores tampoco provocó diferencias estadísticas entre grupos (tabla 3 ).

El consumo acumulado de alimento no arrojó diferencias estadísticamente significativas entre grupos en ninguno de los momentos en que se evaluó (tabla 4).

TABLA 2

Composición proximal del salvado de soja

Componente

Fibra dietaria total (8)

fracción insoluble ( 6):

fracción soluble ( 5):

Proteína cruda (4)

Materia grasa (4)

Cenizas (4)

Humedad (3)

Acido fítico (4)

Hidratos de carbono disponibles \# $\mathrm{g} / 100 \mathrm{~g}$

$56,20 \pm 1,35$

$49,20 \pm 1,34$

$6,94 \pm 0,18$

$7,80 \pm 0,52$

$5,31 \pm 0,41$

$4,12 \pm 0,30$

$3,50 \pm 0,23$

$0,45 \pm 0,04$

22,8 
Los valores del peso corporal de los animales alimentados con las dos dietas no difirieron significativamente en ninguno de los momentos del experimento. Se partió con un peso promedio de $406 \mathrm{~g} \pm 24,9 \mathrm{~g}$ y se constataron los aumentos esperables para el tiempo de tratamiento y la eficiencia de conversión de alimento propia de la edad. Los pesos relativos de los panículos adiposos perigonadales y retroperitoneales no arrojaron diferencias significativas entre los grupos a los $30 \mathrm{ni}$ a los 60 días (tabla 4).

En el grupo $\mathrm{S}$, el peso relativo del hígado fue significativamente menor que en el grupo $\mathrm{C}$ en los animales sacrificados a los 30 y a los 60 días de tratamiento (tabla 4).

El contenido de lípidos totales, colesterol y triacilgliceroles hepáticos fue significativamente inferior en el grupo $\mathrm{S}$ respecto al $\mathrm{C}$, tanto en los animales sacrificados al cabo de los 30 como de los 60 días. La disminución fue de un $30 \%$ a los 30 días y de más de un $50 \%$ a los 60 días de tratamiento (tabla 5).

\section{DISCUSIÓN}

La comprobación de la existencia de ambos tipos de fibra -soluble e insoluble- en el salvado de la soja, le confiere trascendencia nutricional dado que su empleo como componente de alimentos funcionales, otorgaría la posibilidad de obtener simultáneamente los efectos tanto de una fracción como de la otra.

La combinación de los efectos característicos de las fibras insolubles, aumento de la velocidad de vaciamiento intestinal, de la humedad y el volumen de la materia fecal y de la eliminación de sales y ácidos biliares, con los de las fibras solubles -aumento de la viscosidad del contenido gástrico, de la capacidad de saciación y de la

\section{TABLA 3}

Colesterolemia total, triacilglicerolemia (TAG), glucemia basal y glucemia 120 minutos, (mmol/l)

Día

0
30
60

\section{CELULOSA}

SOJA

CELULOSA

SOJA

Colesterolemia

TAG

Glucemia

Glucemia 120

$$
\begin{array}{cc}
3,08 \pm 0,13 & 3,90 \pm 0,16^{*} \mathrm{a} \\
2,30 \pm 0,47 & 3,04 \pm 0,45^{*} \\
9,00 \pm 0,72 & 9,10 \pm 0,44 \\
9,61 \pm 0,66 & 9,11 \pm 0,88
\end{array}
$$$$
3,26 \pm 0,13 b
$$$$
3,95 \pm 0,05^{*} \mathrm{a}
$$$$
3,49 \pm 0,23 \mathrm{~b}
$$$$
2,65 \pm 0,39
$$$$
3,41 \pm 0,35 * *
$$$$
2,82 \pm 0,75
$$$$
8,85 \pm 0,61
$$$$
9,27 \pm 0,61
$$$$
9,05 \pm 0,44
$$

$8,55 \pm 0,89$

$8,72 \pm 0,76$

$8,44 \pm 0,77$

* indica diferencia significativa con respecto al día 0

Letras diferentes indican diferencia significativa entre dietas

\section{TABLA 4}

\section{Consumo acumulado de alimento (g), peso corporal (g), pesos relativos de panículos adiposos retroperitoneales (RP), perigonadales (PG) y de hígado}

\begin{tabular}{lcccc} 
& \multicolumn{2}{c}{ 30 días } & \multicolumn{2}{c}{ 60 días } \\
& CELULOSA & SOJA & CELULOSA & SOJA \\
C. acumulado & $748,0 \pm 40,7$ & $687,5 \pm 35,7$ & $1049,0 \pm 39,6$ & $1143,0 \pm 79,0$ \\
Peso corporal & $431,5 \pm 19,0$ & $438,1 \pm 26,2$ & $444,6 \pm 19,8$ & $456,3 \pm 9,0$ \\
Panículos RP & $2,27 \pm 0,31$ & $2,17 \pm 0,45$ & $3,32 \pm 1,01$ & $3,80 \pm 1,15$ \\
Panículos PG & $2,03 \pm 0,38$ & $1,90 \pm 0,37$ & $2,97 \pm 0,53$ & $2,89 \pm 0,77$ \\
Hígados & $4,32 \pm 0,38 \mathrm{a}$ & $3,64 \pm 0,37 \mathrm{~b}$ & $4,59 \pm 0,17 \mathrm{a}$ & $4,07 \pm 0,42 \mathrm{~b}$
\end{tabular}

Letras diferentes indican diferencia significativa entre dietas 
fermentación en el colon, retardo en la absorción de los hidratos de carbono (27) podría resultar beneficiosa en el control del peso corporal y el perfil glucolipídico de personas obesas $(12,28$ - 30) e incluso en la prevención del cáncer de colon $(31,32)$.

En este estudio, la suma de las acciones de los dos tipos de fibra se puso de manifiesto en forma notoria en el perfil lipídico. En el grupo alimentado con la dieta con cascarilla de soja se constató una significativa disminución del nivel de colesterol total sérico. Este hecho es atribuible por un lado a la fracción insoluble -mayoritariamente celulosa y lignina (33) que induce la derivación del colesterol hepático y sérico hacia la síntesis de sales y ácidos biliares promoviendo su eliminación fecal, y por otro a la fracción soluble, la que al ser fermentada en el colon, da origen a ácidos grasos de cadena corta que luego de ser absorbidos por el enterocito producen inhibición de las primeras etapas de la síntesis del colesterol en el hígado $(31,34,35)$.

Asimismo y coincidiendo con otros estudios que reportan disminución de la triacilglicerolemia derivada del consumo de fibras de leguminosas $(36,37)$, el consumo de la cascarilla de la soja atenuó el aumento característico del nivel sérico de los triacilgliceroles que presenta la línea IIMbBeta, tal como se observó en el grupo alimentado con la dieta con celulosa.

Aunque en forma menos significativa, el salvado de soja también tuvo efectos positivos sobre el perfil glucídico al evitar el progresivo aumento de la glucemia basal que habitualmente presentan las ratas de esta línea (19).

La aceptabilidad de la dieta no se modificó por la presencia del salvado de soja, el consumo acumulado de alimentos por parte de ambos grupos no difirió. El incremento de peso de los animales fue el esperado para la edad y el tiempo de duración del experimento. La ausencia de efectos sobre la saciedad probablemente se deba a la composición de la fracción soluble de la fibra del salvado de soja: presenta un alto contenido de ácido glucurónico en su cadena principal semejante a la estructura molecular de las pectinas (13), hecho que le otorga el comportamiento físicoquímico típico de las fibras poco viscosas.

El valor de los lípidos hepáticos hallados en el grupo de animales alimentados con salvado de soja, muy significativamente inferiores a los del grupo testigo, es aún menor que el reportado en estudios sobre el efecto de los fructanos y del almidón resistente tipo 2 tanto en ratas obesas (Zucker fa/fa) como normales (38-40). Nuevamente los resultados son atribuibles a una sumatoria: los efectos prebióticos en el colon por parte de la fracción soluble más las ya mencionadas derivaciones del colesterol hacia la síntesis de sales y ácidos biliares promovidas por la fracción insoluble de la fibra.

Los efectos del salvado de soja evidenciados en este experimento sumados a la demostrada carencia de toxicidad de la fibra de la soja (41) estimulan a profundizar en el estudio de los mismos, y en la dilucidación de los mecanismos de acción. Estudios en humanos obesos y no obesos completarían la información necesaria para considerar el empleo de este residuo industrial, al que actualmente no se le da importancia nutricional ni comercial, en la formulación de alimentos funcionales destinados al mejoramiento del perfil lipídico sérico y hepático sumados a los efectos de promoción del funcionamiento intestinal.

\section{TABLA 5}

\section{Lípidos hepáticos}

\begin{tabular}{|c|c|c|c|c|c|c|}
\hline & \multicolumn{3}{|c|}{30 días } & \multicolumn{3}{|c|}{60 días } \\
\hline & CELULOSA & SOJA & $\Delta \%$ & CELULOSA & SOJA & $\Delta \%$ \\
\hline $\begin{array}{l}\text { Lípidos totales } \\
(\mathrm{g} / 100 \mathrm{~g})\end{array}$ & $1,52 \pm 0,14 \mathrm{a}$ & $0,99 \pm 0,11 b$ & 35,0 & $3,90 \pm 0,77 \mathrm{c}$ & $1,78 \pm 0,59 \mathrm{~d}$ & 54,0 \\
\hline $\begin{array}{l}\text { Colesterol total } \\
(\mathrm{mmol} / 100 \mathrm{~g})\end{array}$ & $0,16 \pm 0,04 \mathrm{a}$ & $0,10 \pm 0,09 b$ & 39,3 & $0,23 \pm 0,08 \mathrm{c}$ & $0,09 \pm 0,04 \mathrm{~b}$ & 58,5 \\
\hline $\begin{array}{l}\text { Triacilgliceroles } \\
(\mathrm{mmol} / 100 \mathrm{~g})\end{array}$ & $0,27 \pm 0,06 \mathrm{a}$ & $0,18 \pm 0,09 \mathrm{~b}$ & 33,2 & $0,93 \pm 0,14 \mathrm{c}$ & $0,44 \pm 0,12 \mathrm{~d}$ & 52,3 \\
\hline
\end{tabular}




\section{RESUMEN}

Se estudiaron los efectos de la cascarilla o salvado de la soja, con alto contenido de fibra dietaria, de tipo insoluble y soluble, sobre la distribución del tejido adiposo, el perfil lipídico sanguíneo y hepático, la glicemia basal y post sobrecarga glucídica, en ratas adultas de la línea $\mathrm{IIMb} / \beta$, obesa y diabética. Ejemplares de 200 días fueron alimentados durante 30 y 60 días con dos dietas con caseinato como fuente proteica y $10 \mathrm{~g} / 100 \mathrm{~g}$ de fibra de salvado de soja (S) o celulosa microcristalina (C). Tanto a los 30 como a los 60 días de tratamiento se constataron niveles de colesterol sérico significativamente menores en el grupo $\mathrm{S}$ con respecto a C. Los valores de $\mathrm{C}$ fueron asimismo superiores a los del inicio del experimento. $\mathrm{La}$ dieta $\mathrm{S}$ atenuó el aumento de los triacilgliceroles séricos, manifestado en el grupo $\mathrm{C}$ y el progresivo aumento de la glucemia basal habitual en estos roedores. Se registró una significativa disminución de los lípidos totales, el colesterol y los triacilgliceroles hepáticos en el grupo S. Se atribuyen estos efectos, a los mecanismos fisiológicos combinados de las fracciones de fibra del salvado de la soja. Se concluye que este producto podría evaluarse en humanos como un potencial componente de alimentos funcionales.

Palabras clave: salvado de soja; alimentos funcionales; obesidad.

\section{Dirigir la correspondencia a:}

Dra.

María Catalina Olguin

Cátedra de Bromatología

Facultad de Ciencias Bioquímicas y Farmacéuticas

Universidad Nacional de Rosario.

Suipacha 531 - 2000 Rosario,

Argentina.

Tel/Fax 543414804598

E-mail: molguin@fbioyf.unr.edu.ar

Agradecimientos: Los autores agradecen el apoyo recibido de la Fundación Alberto J. Roemmers consistente en un subsidio que hizo posible esta investigación y a Wiener Laboratorios SAIC por la donación de equipos para diagnóstico.

\section{BIBLIOGRAFÍA}

1. Schröder H. Protective mechanisms of the Mediterranean diet in obesity and type 2 diabetes $\mathrm{J}$ Nutr Biochem 2007; 18: 149-160.

2. Mermel V.L. Old Paths new directions: the use of functional foods in the treatment of obesity TIFS 2004; (15): 532-540.

3. Obesity: preventing and managing the global epi- demic. Report of a WHO consultation World Health Organization Tech Rep Service, 2000; 894:I 253.

4. World Health Organization Integrated prevention of noncommunicable diseases. Draft global strategy on diet, physical activity and health. 2003.

5. Galisteo M.; Duarte J.; Zarzuelo A. Effects of dietary fibers on disturbances clustered in the metabolic syndrome J Nutr Biochem 2008; (19): 71-84.

6. Jenkins D.J.; Kendall C.; Marchie A.; Jenkins A. Augustin L. et al. Type 2 diabetes and the vegetarian diet. Am J Clin Nutr 2003; 78 (3) 610S-618S.

7. Brown A.C., Hasler C.M. Position of the American Dietetic Association: Functional Foods. J Am Dietetic Assoc 2009 (109), 4: 735-746.

8. Friedman M. \& Brandon D. Nutritional and health benefits of soy proteins J Agric Food Chem 2001; 49 (3): 1069-1086.

9. Thranathan R \& Mahadevamma S. Legumes, a boon to human nutrition TIFS 2003; 14: 507-18.

10. Messina M.J. Legumes and soybeans: overview of their nutritional profiles and health effects. Am J Clin Nutr; 1999;70 (suppl):439S-50S.

11. Anderson J., Johnstone B., Cook Newell M. Meta Analysis of the effects of soy protein intake on serum lipids. New Engl J Med 1995;(333):276-282.

12. Elia M. \& Cummings J.H. Physiological aspects of energy metabolism and gastrointestinal effects of carbohydrates. Eur J Clin Nutr 2007;61(Supp11):S40-S74

13. Takahashi T., Maeda K; Aoyama T; Yamamoto T.; Takamatsu K. Physiological effects of water soluble soybean fiber in rats. Biosci Biotech Bioch 1999;63(8):1340-1345.

14. Knopp R , Superko R., Davidson M., Insull W., Dujovne C., Kwiterovich P.O., Zavoral J., Graham K., O'Connor R. \& Edelman D. Long term blood cholesterol lowering effects of a dietary fiber supplement. Am J Prevent Med 1999;5:18-23.

15. Ipharraguerre I.; Clark J. Soyhulls as an alternative feed for lactating dairy cows: a review. J Dairy Sci 2003;86:1052-1073.

16. Ludden, P., Cecava M.; Hendrix K. The value of soybean hulls as a replacement for corn in beef cattle diets formulated with or without added fat. J Anim Sci 1995;73: 2706-2712.

17. Olguin M.C., Calderari S.A.; Posadas M.; Revelant G. et al Evaluación química y nutricional de un alimento para adultos a base de soja y cereales. Arch Latinoam Nutr 1995;45:187-192.

18. Olguin M.C., Calderari S.A., Zingale M.I., Posadas, M. et al. Effects of dietary fatty acid composition on fat distribution and glucose tolerance of diabetic 
Beta rats. Diabetes Nutr Metab 1998;11:314-319.

19. Festing, M. \& Greenhouse, D. Abbreviated list of inbred strains of rats. Rats News Letter 1992;26,1012.

20. Calderari S.A., González A., Gayol M. Spontaneous hypertriacylgliceridemic obesity and hyperglycemia in an inbred line of rats. Int J Obesity 1987;11,571579.

21. Spence J.T. Challenges related to the composition of functional foods JFCA 2006; 19(1):S4-S6.

22. Vitali D., Vedrina Dragojevic I., Sebecic B. Effects of incorporation of integral raw materials and dietary fibre on the selected nutritional and functional properties of biscuits Food Chem 2009;114:1462-1469

23. Liener I. Implications of antinutritional components in soybean foods. Crit RevFood Sci 1994;34(1):3167.

24. AOAC Official Methods of Analysis (15th Ed) Washington DC Association of Official Analytical Chemists, 1990.

25. Thompson D. \& Erdman J. Phytic acid determination in soybeans. J Food Sci 1982; 47,513-517.

26. Folch J., Lees M., Stanley G.H. A simple method for the isolation and purification of total lipids from animal tissues. J Biol Chem 1957;226:497-509.

27. Queiroz-Monici, K., Costa, G.E., Da Silva, N., Reis, S. \& De Oliveira A. Bifidogenic effect of dietary fiber and resistant starch from leguminous on the intestinal microbiota of rats. Nutrition 2005;21,602608.

28. Slavin J.L. Dietary fiber and body weight Nutrition 2005;21:411-41.

29. Azadbakht L., Kimiagar M., Mehrabi Y., Esmaillzadeh A., Padyab M, Hu F., Willet W. Soy inclusion in the diet improves features of the metabolic symdrome: a randomized crossover study in postmenopausal women. Am J Clin Nutr 2007;85:735-41.

30. Mahalko J., Sandstead H., Johnson L., Inman L., Milne D., Warner R., Haunz E. Effect of consuming fiber from corn bran, soy hulls, or apple powder on glucose tolerance and plasma lipids in type 2 diabetes. Am J Clin Nutr 1984;(39):25-34.

31. Slavin, J.L. Nutritional benefits of soy protein and soy fiber. J Am Diet Assoc 1991; 91,816-819.

32. Pupponen-Pimia R., Aura A., Oksman-Caldentey K., Myllärinen P., Saarella M., Mattila-Sandholm T., Poutanen K. Development of functional ingredients for gut health. TIFS 2002;13:3-11.

33. Redondo-Cuenca A., Villanueva-Suárez M., Rodríguez-Sevilla M., Mateos-Aparicio I. Chemical composition and dietary fibre of yellow and green comercial soybeans (Glycine max). Food Chem 2006;101:1216-1222.

34. Story, J.A. \& Kritchevsky D. Dietary fiber and lipid metabolism. In G.A. Spiller \& R.J. Amen (Eds). Fiber in human nutrition, New York: Plenum Press, 1976;pp 171- 184.

35. Mitamura R., Hara H., Aoyama Y., Takahasi T., Furuta H. Ingestion of water soluble fiber prevents osteopenia and hypercholesterolemia induced by ovariectomy in rats. J Agric Food Chem 2003;51:1085-1089.

36. Rodríguez, R., Jiménez, A., Fernández-Bolaños, J., Guillén, R. \& Heredia, A. Dietary fiber from vegetable products as source of functional ingredientes. TIFS 2006;17,3-15.

37. Guillon F. \& Champ M.J. Carbohydrate fractions of legumes: use in human nutrition and potential for health. Br J Nutr 2002;Suppl 3:293S-306S.

38. López H., Levrat-Verny M., Coudray C., Besson C et al. Class 2 resistant starches lower plasma and liver lipids and improve mineral retention in rats. $\mathrm{J}$ Nutr 2001;131: 1283-1289.

39. Cheng, H.H. \& Lai, M.H. Fermentation of resistant rice starch produces propionate reducing serum and hepatic cholesterol in rats. J Nutr 2000; 130:19911995.

40. Daubioul, C., Rousseau, N., Demeure, R., Gallez, B., Taper, H., Declerck, B. \& Delzenne N. Dietary fructans, but not cellullose decrease triglyceride accumulation in the liver of obese Zucker fa/fa rats. J Nutr 2002;132:967-973.

41. Takahashi, T., Nakamura, A., Kato, M., Mandella, R., Broadmeadow, A. \& Ruckman, S. Soluble soybean fiber: a 3 months toxicity study in rats. Food Chem Toxicol, 2003;41:1111-1122. 